\title{
Sensitivity analysis of multi-objective optimization for solid waste management: A case study of Dares Salaam, Tanzania
}

\author{
Halidi A. Lyeme ${ }^{1,3}$, Allen Mushi ${ }^{2}$ and Yaw Nkansah-Gyekye ${ }^{1}$ \\ ${ }^{1}$ Department of Mathematics, Nelson Mandela African Institution of Science and Technology, Arusha, Tanzania \\ ${ }^{2}$ Department of Mathematics, University of Dar es Salaam, Dar es Salaam, Tanzania \\ ${ }^{3}$ Department of Mathematics, Muslim University of Morogoro, Morogoro, Tanzania
}

Received: 17 December 2016, Accepted: 27 February 2017

Published online: 8 November 2017.

\begin{abstract}
In this study, a sensitivity analysis of a multi-objective optimization model for solid waste management (SWM) for Dar es Salaam city in Tanzania is considered. Our objectives were to identify the most sensitive parameters and effect of other input data to the model output. Five scenarios were considered by varying their associated parameter values. The results showed that the decrease of total cost for the SWM system in all scenarios was observed compared to the baseline solution when the single landfill was considered. Furthermore, the analysis shows that the variable cost parameter for the processing facilities is very sensitivity in such a way that if you increase the variable cost then, there is a rapid increase of total cost for the SWM system and the vice versa is true. The relevant suggestions to the decision makers were also discussed.
\end{abstract}

Keywords: Sensitivity analysis, optimum path, baseline solution, sensitive parameter, cost for SWM system.

\section{Introduction}

An important aspect of the model solution phase is sensitivity analysis. It is designed to study the effect of changes in the parameters of the model on the optimum solution. Such analysis is regarded as an integral part of the solution of any real life problem. It gives a model a dynamic characteristic that allows a researcher to study the behavior of the optimal solution as a result of making changes in the model parameters. The ultimate objective of the analysis is to obtain information about possible new optimum solutions corresponding to changes in the parameters with minimal additional computations [5].

Sensitivity analysis is an analysis that determines how sensitive the model solution to any change in a parameter while keeping other parameters constant. Sensitivity analysis is practical since it informs the user of the model on how the solution value do depend on every known value of the model. It permits him an idea of how much room he has for every variable to go adversely. Sensitivity analysis is useful when the values of the problem parameters are unknown accurately $[2]$.

Mathematical models are applied in various complicated economic, engineering, social, physical and environmental problems [3]. The model development process includes some logical steps, one of which is the determination of the input information (parameters) of the model which are most powerful in the model solution. A post-optimality analysis of 
these input information is very significant to the model verification and will also serve for the forthcoming study [4].

Modelers may conduct the post-optimality analyses for a number of rationale including the requirements to determine: (1) which input information need further study to give strength to the knowledge base, so that decreasing the solution variability; (2) which input information are irrelevant and can be removed from the final model; (3) which parameters cause the variational to the model solution; (4) which parameters are most highly correlated with the solution; and (5) once the model is in production use, what consequence results from altering a given input information. There are many diverse ways of conducting post-optimality analyses; however, in answering these questions the various analyses may not generate the same results [6].

In general, post-optimality analyses are conducted by defining the model, its decision variables and parameters, assigning probability density functions to every input data, generating an input matrix through a suitable random sampling method, calculating an output vector, and evaluating the effects and relative importance relationship of every input or output [8].

The ranges within which the values of the parameters can change before the optimal solution is no longer optimal give an indication of how critically the decisions implied by the optimal solution depend on the accuracy of the specified parameters. Sensitivity analysis considers the effect on the optimal solution by changing the objective function coefficients and also by changing the right hand side coefficients of the constraints [9]. At times certain known values may serve as the managerial decisions. In this case, the choice of the parameter values may be the main issue to be researched and this can be done through post-optimality analysis.

Therefore, it is possible to explain various inclinations that the decision makers could make due to the criteria used, and how they influence the study solutions [1].

The purpose of this paper is to perform the sensitivity analysis for the multi-objective optimization model for solid waste management proposed in section 3. The sensitivity of landfill site alternative, fixed cost for the processing facilities, variable coast, transportation cost and recycling limitation were tested by varying their associated parameters. The sensitivity of separation plant was also tested by considering what will happen to the optimal solution if the cost for sorting was not included.

\section{Multi-objective optimization model}

In this section, we briefly described the model proposed by [7], their model has three objective functions as shown in equation (1), (2) and (3).

The first one deal with the total cost minimization, which contains the cost for transportation, recycling, separation, composting, incineration, and recovered from the disposal of waste. The costs for every operating facility and capital costs are also included in this objective function. The second objective function deal with the minimization of total environment impact, such as greenhouse gases (GHG) emissions which includes carbon and methane emissions due to recycling, composting, incineration and waste disposal to the landfill. The last objective function minimizes the final waste disposed to the landfill from all separation facilities. The multi-objective model for solid waste management proposed by [7], is given below; 


$$
\begin{aligned}
& \left(\sum_{j=1}^{J} F R_{j} R_{j}+\sum_{h=1}^{H} F M_{h} S_{h}+\sum_{g=1}^{G} F P_{g} U_{g}+\sum_{k=1}^{K} F S_{k} V_{k}+\right. \\
& \sum_{l=1}^{L} F C_{l} W_{l}+\sum_{m=1}^{M} F E_{m} X_{m}+\sum_{n=1}^{N} F L_{n} Y_{n}+\sum_{k=1}^{K} \sum_{i=1}^{I} V S_{k} A W_{i k} \\
& +\sum_{j=1}^{J} \sum_{k=1}^{K} V R_{j} A S_{k j}+\sum_{h=1}^{H} \sum_{k=1}^{K} V M_{h} B S_{k h}+\sum_{g=1}^{G} \sum_{k=1}^{K} V P_{g} P S_{k g} \\
& M i n Z_{1}=\left\{\begin{array}{l}
+\sum_{l=1}^{L} \sum_{\substack{k=1 \\
I}}^{K} V C_{l} D S_{k l}+\sum_{m=1}^{M} \sum_{\substack{k=1 \\
K}}^{K} V E_{m} E S_{k m}+\sum_{n=1}^{N} \sum_{k=1}^{K} V L_{n} L S_{k n}
\end{array}\right. \\
& \text { MinZ }_{1}= \begin{cases}l=1 & \begin{array}{ll}
l=1 \\
+
\end{array} \sum_{i=1}^{I} \sum_{k=1}^{K} T S_{i k} A W_{i k}+\sum_{k=1}^{K} \sum_{j=1}^{J} T R_{k j} A S_{k j}+\sum_{k=1}^{K} \sum_{h=1}^{H} T M_{k h} B S_{k h}\end{cases} \\
& +\sum_{k=1}^{K} \sum_{g=1}^{G} T P_{k g} P S_{k g}+\sum_{k=1}^{K} \sum_{l=1}^{L} T C_{k l} D S_{k l}+\sum_{k=1}^{K} \sum_{m=1}^{M} T E_{k m} E S_{k m} \\
& +\sum_{k=1}^{K} \sum_{n=1}^{N} T L_{k n} L S_{k n}+Q_{j} \sum_{k=1}^{K} \sum_{j=1}^{J} A S_{k j}+M_{h} \sum_{k=1}^{K} \sum_{h=1}^{H} B S_{k h} \\
& +P_{g} \sum_{k=1}^{K} \sum_{g=1}^{G} P S_{k g}+C_{l} \sum_{k=1}^{K} \sum_{l=1}^{L} D S_{k l}+E_{m} \sum_{k=1}^{K} \sum_{m=1}^{M} E S_{k m} \\
& \operatorname{Min} Z_{2}=\left\{\begin{array}{l}
G_{j}^{G H E} \sum_{k=1}^{K} \sum_{j=1}^{J} A S_{k j}+B_{h}^{G H E} \sum_{k=1}^{K} \sum_{h=1}^{H} B S_{k h}+D_{g}^{G H E} \sum_{k=1}^{K} \sum_{g=1}^{G} P S_{k g} \\
+E_{l}^{G H E} \sum_{k=1}^{K} \sum_{l=1}^{L} D S_{k l}+F_{m}^{G H E} \sum_{k=1}^{K} \sum_{m=1}^{M} E S_{k m}+H_{n}^{G H E} \sum_{k=1}^{K} \sum_{n=1}^{N} L S_{k n}
\end{array}\right. \\
& \operatorname{Min} Z_{3}=\sum_{k=1}^{K} \sum_{n=1}^{N} L S_{k n}
\end{aligned}
$$

subject to the constraints:

$$
\begin{gathered}
\sum_{i=1}^{I} \sum_{k=1}^{K} A W_{i k}=A_{i}, \text { for } i=(1, . ., I) \\
\sum_{k=1}^{K} \sum_{j=1}^{J} A S_{k j}=\sum_{i=1}^{I} \sum_{k=1}^{K} \alpha_{1} A W_{i k} \\
\sum_{k=1}^{K} \sum_{h=1}^{H} B S_{k h}=\sum_{i=1}^{I} \sum_{k=1}^{K} \alpha_{2} A W_{i k} \\
\sum_{k=1}^{K} \sum_{g=1}^{G} P S_{k g}=\sum_{i=1}^{I} \sum_{k=1}^{K} \alpha_{3} A W_{i k}
\end{gathered}
$$




$$
\begin{gathered}
\sum_{k=1}^{K} \sum_{l=1}^{L} D S_{k l}=\sum_{i=1}^{I} \sum_{k=1}^{K} \beta A W_{i k} \\
\sum_{k=1}^{K} \sum_{m=1}^{M} E S_{k m}=\sum_{i=1}^{I} \sum_{k=1}^{K} \gamma A W_{i k} \\
\sum_{k=1}^{N} S_{k n}=\sum_{i=1}^{I} \sum_{k=1}^{K}\left(1-\alpha_{1}-\alpha_{2}-\alpha_{3}-\beta-\gamma\right) A W_{i k} \leq C L_{n} Y_{n}, \text { for } n=(1, . ., N) \\
\sum_{k=1}^{K} \sum_{h=1}^{I} A W_{i k} \leq C S_{k} V_{k}, \text { for } k=(1, . ., K) \\
\sum_{k=1}^{K} P S_{k g} \leq C R_{g} U_{g}, \text { for } g=(1, . ., G) \\
\sum_{k=1}^{K} A S_{k j} \leq C E_{m} X_{m}, \text { for } R_{j}, \text { for } j=(1,2, \ldots, J) \\
B S_{k h} \leq C R_{h} S_{h}, \text { for } h=(1, . . ., H)
\end{gathered}
$$




$$
\begin{gathered}
U_{g}=0 \text { or } 1, \text { for } g=(1,2, \ldots, G) \\
V_{k}=0 \text { or } 1, \text { for } k=(1,2, \ldots, K) \\
W_{l}=0 \text { or } 1, \text { for } l=(1,2, \ldots, L) \\
X_{m}=0 \text { or } 1, \text { for } m=(1,2, \ldots, M) \\
Y_{n}=0 \text { or } 1, \text { for } n=(1,2, \ldots, N) \\
\left\{\begin{array}{l}
A W_{i k} \geq 0, A S_{k j} \geq 0, B S_{k h} \geq 0, P S_{k g} \geq 0, D S_{k l} \geq 0, \\
E S_{k m} \geq 0, L S_{k n} \geq 0 \forall i, j, h, g, k, l, m, n
\end{array}\right.
\end{gathered}
$$

\section{Sensitivity analysis of the model}

The input data to the model is the key point for the sensitivity analysis of any model. Using the sensitivity analysis based on the optimal solution, the effect of the parameters change can be clearly exemplified. In this section, the sensitivity analysis of landfill site alternative, fixed and variable cost for the waste processing facilities, transportation cost and recycling limitation will be tested by varying their associated parameters. The sensitivity of separation plant was also tested by considering what will happen to the optimal solution if the cost for sorting was not included.

\subsection{Landfill site alternative analysis}

In this scenario, two hypothetical alternative landfills are considered. Both have identical operating costs, construction and flow capacity. One landfill is assumed to be located in the northeastern part of the city (Goba) and the other landfill in the southeastern part of the city (Kisarawe). The major assumption in this scenario is that the land for both landfills is available for construction at any time and thus the Municipal Authority has preserved this land. The overall point of this scenario is to analyze the impact of landfill options on landfill management in the city, in particular, the impact of construction and operating costs of the new landfills and their locations. If new landfills are relatively cheap to operate then this will reduce diversion needs. If landfills are located faraway, and costly then this will raise diversion needs.

Let us add the two landfill sites (Goba and Kisarawe) to the model formulated in section 3. The transportation cost from separation plants to the landfills are given in Table 1. 
Table 1: Transportation Costs (Tsh/ton) from Separation to Landfills

\begin{tabular}{l|lll}
\hline \multirow{2}{*}{$\begin{array}{l}\text { SEPARATION } \\
\text { PLANT }\end{array}$} & \multicolumn{3}{|c}{ LANDFILL } \\
\cline { 2 - 4 } Ilala & 11625 & 21750 & 31425 \\
Upanga & 20175 & 20025 & 31200 \\
Segerea & 15000 & 19800 & 31650 \\
Buguruni & 17025 & 20475 & 29400 \\
Ukonga & 15450 & 23250 & 30825 \\
Pugu & 8700 & 24975 & 32400 \\
Kawe & 27600 & 9975 & 42000 \\
Bunju & 32100 & 15450 & 55650 \\
Kigogo & 18150 & 18750 & 30450 \\
Tandale & 21225 & 17325 & 33075 \\
Mbezi & 12825 & 13800 & 37575 \\
Kibamba & 23850 & 15900 & 47475 \\
Kimara & 17250 & 15075 & 34800 \\
Ubungo & 20100 & 13500 & 36150 \\
Mbagala & 18375 & 23175 & 25950 \\
Kibondemaji & 21075 & 28050 & 22425 \\
Mjimwema & 34350 & 41775 & 7575 \\
Vijibweni & 37425 & 44850 & 9000 \\
Chang'ombe & 17025 & 21600 & 27975 \\
Mtoni & 18225 & 22875 & 24000 \\
\hline
\end{tabular}

The sensitivity analysis of the model was tested by including the two landfills with their fixed and variable costs. In the first case we include one landfill (Goba) so that the model has two landfills. In the second case, we include two landfills (Goba and Kisarawe) so that the model has three landfills. The result shows the decrease of total cost for SWM for both cases by $2.7 \%$ (Tsh. 73,056,058.75) and 4\% (Tsh. 71,233,941) respectively compared to baseline solution when the single landfill was considered. The different optimum path for allocation of the waste amount from separation plants to the landfill for the two cases was also observed as shown in Table 2 and Table 3 respectively. It is worth mentioning that the case with three landfills gives the minimum cost for the SWM system in the case study area, and therefore the decision makers should adopt this case.

Table 2: Waste Amount (ton) Flow from Separation to Landfills

\begin{tabular}{l|ll}
\hline \multirow{2}{*}{$\begin{array}{l}\text { SEPARATION } \\
\text { PLANT }\end{array}$} & \multicolumn{2}{|c}{ LANDFILL } \\
\cline { 2 - 3 } & KINYAMWEZI & GOBA \\
\hline Ilala & 66 & 48.6 \\
Upanga & & \\
Segerea & 64.9 & \\
Buguruni & 66 & \\
Ukonga & 66 & \multicolumn{2}{c}{ Continued on next page }
\end{tabular}




\begin{tabular}{l|ll}
\multicolumn{2}{c}{ Table 2 - Continued from previous page } \\
\hline \multirow{2}{*}{$\begin{array}{l}\text { SEPARATION } \\
\text { PLANT }\end{array}$} & \multicolumn{2}{|c}{ LANDFILL } \\
\cline { 2 - 3 } Pugu & 66 & 66 \\
Kawe & & 10.3 \\
Bunju & & 44.5 \\
Kigogo & 21.5 & 66 \\
Tandale & & \\
Mbezi & 66 & \\
Mbagala & 66 & \\
Mjimwema & 52.6 & \\
Vijibweni & 33 & \\
Chang'ombe & 66 & \\
Mtoni & 66 & \\
\hline
\end{tabular}

Table 3: Waste Amount (ton) Flow from Separation to Landfills

\begin{tabular}{|c|c|c|c|}
\hline \multirow{2}{*}{$\begin{array}{l}\text { SEPARATION } \\
\text { PLANT }\end{array}$} & \multicolumn{3}{|c|}{ LANDFILL } \\
\hline & KINYAMWEZI & GOBA & KISARAWE \\
\hline Ilala & 66 & & \\
\hline Upanga & & 47.5 & \\
\hline Segerea & 66 & & \\
\hline Buguruni & 66 & & \\
\hline Ukonga & 66 & & \\
\hline Pugu & 66 & & \\
\hline Kawe & & 66 & \\
\hline Bunju & & 10.3 & \\
\hline Kigogo & 66 & & \\
\hline Tandale & & 66 & \\
\hline Mbezi & 66 & & \\
\hline Mbagala & 66 & & \\
\hline Mjimwema & & & 52.6 \\
\hline Vijibweni & & & 33 \\
\hline Chang'ombe & 66 & & \\
\hline Mtoni & 66 & & \\
\hline
\end{tabular}

\subsection{Fixed cost analysis}

Fixed costs in processing facilities are considered as the cost for construction or opening of the processing facilities for the SWM. They are directly associated by the waste management alternatives and have an influence on the total cost of 
the SWM system. The sensitivity analysis of the model to the fixed costs was tested by assuming the existence of these processing facilities, that is, there is no cost for constructing or opening of these processing facilities.

The result shows that there is a rapid decrease of the total cost to Tsh 44,689,260.25 which implies that the fixed cost for opening processing facilities has a great impact on the SWM system. This shows that the total cost for the SWM system is viable if the fixed cost of constructing or opening of the processing facilities is not included in the model. Thus, the fixed cost will result in a higher total cost at the beginning of constructing the waste management alternatives while the total cost for the SWM system will decrease if there is a continuation of using previously constructed processing facilities.

Moreover, the sensitivity of separation plant was tested considering if waste sorting is done at the source and fixed cost for separation plant become zero. The result shows that there is a decrease of total cost for the SWM system to Tsh. $61,358,762.25$. Therefore, introducing a new law which enforces society to sort waste at the source will reduce the total cost for the SWM system.

\subsection{Processing cost analysis}

Variable operating cost in processing facilities are considered as cost sinks to the model. They are not ultimate waste management alternatives. However, they are necessary for launching other waste management options like separation, composting, incineration, recycling and landfill. The sensitivity of the model to the processing costs was tested by changing the variable operating costs of processing facilities as shown in Table 4.

Table 4: Different Cases for the Changing of the Variable Cost

\begin{tabular}{llll}
\hline & Case 1 & Case 2 & Case 3 \\
\hline Variable Cost & Tsh. 55,000 & Tsh. 65,000 & Tsh. 75,000 \\
Total SWM Cost & Tsh. 13,806,058.75 & Tsh. 71,233,941.25 & Tsh. 156,273,941 \\
\hline
\end{tabular}

Decreasing the variable operating cost of processing facilities to Tsh 55,000 per ton (Case 1) gave the same optimum path (waste allocation to processing facilities) as that of Case 2 at the variable operating cost of Ths 65,000 per ton with decreasing the total cost for the SWM system. Increasing its cost to Tsh 75,000 per ton (Case 3) yielded the same optimum path of case 2 with increasing the total cost for the SWM system.

As far as the variable cost for processing facilities is concerned, a change in the variable cost seems to bear the most significant change in the total cost for the SWM system. An increase of variable cost by Tsh. 10,000 has increased the total cost for the SWM system by $120 \%$. In the other hand if we decrease the variable cost by Tsh. 10,000, there is the decrease of $80.5 \%$. This means that the variable cost parameter is very sensitive therefore care should be taken whenever the decision makers plan for the SWM alternatives. 


\subsection{Transportation cost analysis}

In this scenario, we consider the addition of the model assumption started as if all generated solid waste are transferred to the nearest separation plants at the expense of their generators what will happen to the total cost of the SWM system. The sensitivity of this parameter was tested and reveal the decrease of total cost for the SWM system to Tsh. 55,529,103.63 which is $22.1 \%$ decrease compared to the base optimal solution. The optimum path for solid waste allocation to different processing facilities and landfills remain unchanged (same as shown in Table 3). This indicates that the participation of the community to the SWM system reduces the cost. Therefore, the decision makers should consider this option in order to have an optimum SWM system cost.

\subsection{Recycling limitations analysis}

In this scenario, we consider the sensitivity of changing the fraction of recycling materials to the model solution. Three cases were considered in this scenario; in the first case we reduced the fraction of plastic material by $2 \%$ while in the second case we increased the fraction of plastic material by $2 \%$. The results show the inverse relationship between fraction of plastic material and total cost for the SWM system in both two cases. In the first case, there is an increase of total cost for the SWM system by $18.1 \%$ (Tsh 98,502,733.7) while in other case there is a decrease of total cost for the SWM system by $45.9 \%$ (Tsh 12,198,882) compared to the base case.

In the third case, we increased by $1 \%$ the fraction of recycling materials for plastic, metal and paper respectively. The output of this case shows that there is a decrease of the total cost for the SWM system by $40.9 \%$ (Tsh 18,881,233.2) compared to the base case. It should be noted that the increase of the fraction of recycling materials is more significant than that of reducing it. The reason is that the final waste to the landfill will be reduced due to the increased fraction of recycling materials and finally, the GHG emissions will also be reduced.

\section{Conclusion}

In this section, the sensitivity analysis of the multi-objective optimization model proposed in chapter 3 has been considered. Different scenarios and cases were analyzed and discussed with relevant suggestions to the decision makers.

The sensitivity of landfill alternatives was tested, first by incorporating one landfill and the other by incorporating two landfills. The decrease of total cost for the SWM system for both cases by $2.7 \%$ and $4 \%$ respectively were observed compared to the baseline solution when the single landfill was considered. The decrease of total cost for the SWM system by $22.1 \%$ compared to the base optimal solution was also observed when the sensitivity analysis of transportation cost was tested by assuming that if the transportation cost from the source to separation plant will be taken care by the waste generators.

The sensitivity analysis of fixed cost of the processing facilities was also tested and generally shows the decrease of the total cost for the SWM system. In addition to that, the sensitivity of separation plant was tested by considering wastes sorting at the source and result shows promising decreasing of the total cost for the SWM system. Therefore, introducing the law which will enforce the society to sort the waste at the sources will reduce the total cost for the SWM system. 
Furthermore, the analysis shows that the variable cost parameter for the processing facilities is very sensitivity in such a way that if you increase the variable cost then, there is up rapidly increase of total cost for the SWM system and the vice versa is true. Therefore, great care should be taken whenever the decision makers plan for the SWM system alternative.

Finally, the sensitivity analysis of the fraction of the recycling materials was also tested and the output shows the decrease of the total cost for the SWM system if there is a reasonable increase of the fraction of the recycling materials. Therefore, the increase of the fraction of the recycling materials will reduce the final waste to the landfills and hence, will reduce the GHG emissions.

\section{Acknowledgements}

The authors would like to thank the Government of the Republic of Tanzania through the Commission for Science and Technology (COSTECH) for the full financial support of this study.

\section{Competing interests}

The authors declare that they have no competing interests.

\section{Authors' contributions}

All authors have contributed to all parts of the article. All authors read and approved the final manuscript.

\section{References}

[1] Babalola, M. A. (2015). A multi-criteria decision analysis of waste treatment options for food and biodegradable waste management in japan. Environments. 2(4): 471-488.

[2] Chinneck, J. W. (2006). Practical optimization: a gentle introduction. Systems and Computer Engineering), Carleton University, Ottawa. http://www. sce. carleton. ca/faculty/chinneck/po. html.

[3] Hamby, D. (1994). A review of techniques for parameter sensitivity analysis of environmental models. Environmental monitoring and assessment. 32(2): 135-154.

[4] Helton, J., Iman, R., Johnson, J. and Leigh, C. (1986). Uncertainty and sensitivity analysis of a model for multicomponent aerosol dynamics. Nuclear technology. 73(3): 320-342.

[5] Hillier, F. S. (2012). Introduction to operations research. Tata McGraw-Hill Education.

[6] Iman, R. L. and Helton, J. C. (1988). An investigation of uncertainty and sensitivity analysis techniques for computer models. Risk analysis. 8(1): 71-90.

[7] Lyeme, H. A., Mushi, A. and Nkansah-Gyekye, Y. (2016). Multi-objective optimization model formulation for solid waste management in dar es salaam, tanzania. Asian Journal of Mathematics and Applications. 2017.

[8] Provsek, A. and Leskovar, M. (2015). Use of fast fourier transform for sensitivity analysis. http:cdn.intechopen.com/pdfswm/48177.pdf. Accessed on 10 November 2016.

[9] Vanderbei, R. J. et al. (2015). Linear programming. Springer. 